\title{
COMMENTARY
}

\section{Airway management disasters in the ICU - lessons learned?}

\author{
Christian Byhahn ${ }^{1, *}$ and Erol Cavus ${ }^{2}$ \\ See related research by Noppens et al., http://ccforum.com/content/16/3/R103
}

\begin{abstract}
The C-MAC video laryngoscope substantially reduced poor glottic views and increased intubation success in ICU patients with at least one predictor for difficult intubation. However, all video-assisted and optical intubation devices have different view angles, thus producing different images with particular distortion, and even experts in 'old-fashioned' airway management need a substantial level of training with a certain device before using it safely and successfully in critical situations and patients. Video laryngoscopes, regardless of a particular brand or device, cannot be used intuitively - they require expert skills and routines to be turned into a life-saving airway management tool.
\end{abstract}

In recent years, video-assisted intubation devices have flooded theaters, and, to a lesser extent, are also being used in ICUs. But can such new technology really contribute to making tracheal intubation in the ICU less life-threatening, or even more life-saving? Noppens and colleagues [1], in a study just published in Critical Care, evaluated the efficacy of the C-MAC video laryngoscope [2] for endotracheal intubation in an ICU managed by anesthesiologists and compared the results to those obtained during a previous period when conventional direct laryngoscopy was used. In patients with at least one predictor for difficult intubation, poor glottic views (Cormack and Lehane grades III and IV) were reduced from $38 \%$ to $19 \%$, and intubation success on the first attempt increased from $56 \%$ with direct laryngoscopy to $79 \%$ when the C-MAC video laryngoscope was used. Even in all patients, the incidence of at least two

*Correspondence: c.byhahn@em.uni-frankfurt.de

'Department of Anesthesiology, Intensive Care Medicine, and Pain Therapy,

JW Goethe-University Medical School, Frankfurt, Germany

Full list of author information is available at the end of the article intubation attempts was reduced from 7\% with direct laryngoscopy to $3 \%$ with video laryngoscopy. Though this was not statistically significant, halving this incidence may have substantial clinical impact. Although the overall intubation success was $100 \%$ in either group, successful tube placement in the trachea on the first attempt is crucial in ICU patients with poor tolerance to apnea.

After implementation of a video laryngoscope, however, intubations were more often performed by less experienced physicians. This trend has to be considered carefully. Even if one reason may be that younger physicians are more open to the newer technique, it must be guaranteed that physicians' experience is not substituted solely by technical equipment. Similarly, the use of endoscopic intubation was reduced substantially in the intervention phase ( $4 \%$ versus $13 \%$ ), so experience with such devices may decrease, causing problems if video laryngoscopy is not possible or is contraindicated.

How do these data influence our future airway management strategies in the ICU? Video laryngoscopy for every patient? Sparing such devices for the really difficult cases? To answer these questions it needs to be emphasized that all video-assisted and optical intubation devices have different view angles, thus producing different images with particular distortions, and even experts in 'old-fashioned' airway management need a substantial level of training with a certain device before using it safely and successfully in critical situations and patients [3]. Video laryngoscopes, regardless of a particular brand or device, cannot be used intuitively - they require expert skills and routines to be tamed and turned into life-saving tools. Tube placement can turn out to be difficult and even impossible, despite the excellent glottic exposure video laryngoscopes most often provide. The reason is that the operator's eye is no longer in a straight optical and anatomical - line with the larynx, but at the tip of the laryngoscope blade. Optical and anatomical axes divert, and tube placement is sometimes difficult or fails [4]. Becoming alert to these potential problems associated with video laryngoscopy and constantly improving one's technical skills to overcome such pitfalls 
can only be achieved when such devices are used on a daily basis and in routine patients.

Apart from the primary aim of the study - evaluating video laryngoscopy - it needs to be highlighted that Noppens and colleagues [1] routinely used capnography to verify correct tube placement after intubation. Capnography has become a mandatory requirement in any operating room, but its use in the ICU seems still to be somewhat dowdy. As of 2010, only $25 \%$ of ICUs in the UK and the Republic of Ireland used capnography to confirm correct tube placement after intubation [5]. Such refusal may produce catastrophic outcomes: data collected from all UK National Health Service hospitals over a period of one year in 2008/2009 revealed that $61 \%$ of adverse events during airway management in the ICU resulted in death or permanent brain damage. In $74 \%$ of such fatal cases no capnography was used [6]!

Doubtless, video laryngoscopy is a helpful tool in the hands of experienced physicians, but should not be considered a bailout procedure for beginners in airway management. And as attractive and simple video laryngoscopy appears, we must always remind ourselves to maintain our skills in endoscopic intubation. Lessons learned?

\section{Competing interests}

Both authors receive continuous material support for clinical studies from Karl Storz GmbH \& Co. KG, the manufacturer of the C-MAC video laryngoscope.

This potential conflict of interest, however, did not bias this work.

\section{Author details}

'Department of Anesthesiology, Intensive Care Medicine, and Pain Therapy, JW Goethe-University Medical School, Frankfurt, Germany. ${ }^{2}$ Department of Anaesthesiology and Intensive Care Medicine, University Hospital SchleswigHolstein, Campus Kiel, Kiel, Germany.

Published: 29 October 2012

\section{References}

1. Noppens RR, Geimer S, Eisle N, David M, Piepho T: Endotracheal intubation using the $\mathrm{C}^{-M A C}{ }^{\otimes}$ video laryngoscope or the Macintosh laryngoscope: a prospective, comparative study in the ICU. Crit Care 2012, 16:R103.

2. Cavus E, Kieckhaefer J, Doerges V, Moeller T, Thee C, Wagner K: The C-MAC videolaryngoscope: first experiences with a new device for videolaryngoscopy-guided intubation. Anesth Analg 2010, 110:473-477.

3. Levitan RM, Heitz JW, Sweeney M, Cooper RM: The complexities of tracheal intubation with direct laryngoscopy and alternative intubation devices. Ann Emerg Med 2011, 57:240-247.

4. Byhahn C, Iber T, Zacharowski K, Weber CF, Ruesseler M, Schalk R, Meininger D: Tracheal intubation using the mobile C-MAC video laryngoscope or direct laryngoscopy for patients with a simulated difficult airway. Minerva Anestesiol 2010, 76:577-583.

5. Georgiou AP, Gouldson S, Amphlett AM: The use of capnography and the availability of airway equipment on Intensive Care Units in the UK and the Republic of Ireland. Anaesthesia 2010, 65:462-467.

6. Cook TM, Woodall N, Harper J, Benger J; Fourth National Audit Project: Major complications of airway management in the UK: results of the Fourth National Audit Project of the Royal College of Anaesthetists and the Difficult Airway Society. Part 2: intensive care and emergency departments. Br J Anaesth 2011, 106:632-642.

doi:10.1186/cc11641

Cite this article as: Byhahn C, Cavus E: Airway management disasters in the ICU - lessons learned? Critical Care 2012, 16:162. 